\title{
The TRIple PLunger for EXotic beams TRIPLEX for excited-state lifetime measurement studies on rare isotopes
}

\author{
H. Iwasaki ${ }^{\mathrm{a}, \mathrm{b}}$, A. Dewald ${ }^{\mathrm{c}}$, T. Braunroth ${ }^{\mathrm{c}}$, C. Fransen ${ }^{\mathrm{c}}$, D. Smalley ${ }^{\mathrm{a}}$,

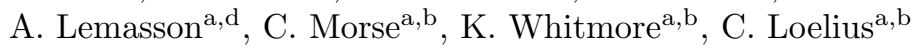 \\ ${ }^{a}$ National Superconducting Cyclotron Laboratory, Michigan State University, East Lansing, \\ Michigan 48824, USA \\ ${ }^{b}$ Department of Physics and Astronomy, Michigan State University, East Lansing, \\ Michigan 48824, USA \\ ${ }^{c}$ Institut für Kernphysik der Universität zu Köln, D-50937 Cologne, Germany \\ ${ }^{d}$ GANIL, CEA/DSM-CNRS/IN2P3, Bd Henri Becquerel, BP 55027, F-14076 Caen Cedex \\ 5, France
}

\begin{abstract}
A new device, the TRIple PLunger for EXotic beams (TRIPLEX), has been developed for lifetime measurement studies with rare isotope beams. This plunger device holds up to three metal foils in the beam path and facilitates the recoil distance Doppler-shift technique to measure lifetimes of nuclear excited states in the range of $1 \mathrm{ps}-1 \mathrm{~ns}$. The unique design allows independent movement of the target and second degrader with respect to a fixed first degrader in between, enabling advanced experimental approaches, such as the differential recoil distance method and the double recoil distance method. The design and control of the device are presented in this paper, together with simulated performances of the new applications. As an example of actual experiments, results from the lifetime measurement of the neutron-rich ${ }^{17} \mathrm{C}$ isotope performed at the National Superconducting Cyclotron Laboratory are shown.

Keywords: Plunger, Lifetimes, Recoil distance Doppler-shift method, Rare isotope beams
\end{abstract}

Preprint submitted to Nuclear Instruments and Methods in Physics Research Section AAugust 28, 2015

(C) 2015. This manuscript version is made available under the Elsevier user license http://www.elsevier.com/open-access/userlicense/1.0/ 


\section{Introduction}

2 In this paper, we report on the development of a new plunger device for life3 time measurements of exotic nuclei based on the Recoil Distance Doppler-Shift 4 method (RDDS) using intermediate-energy rare isotope beams. Excited-state lifetimes have been extensively studied to determine transition rates among nuclear levels which offer useful information to quantify deformation of atomic nuclei, characterize collective modes, and understand wave functions of the states. Experimental studies to determine lifetimes have been performed with a variety of reaction mechanisms involving stable and rare isotope beams at low and relativistic energies. A recent review of the related works can be found in Ref. [1].

The limiting factors for precision in recoil distance studies are predominantly the experimental yields in $\gamma$-ray spectra and the necessity for measurements with various distance settings. These are particularly relevant for experiments with rare isotope beams when nuclei of interest are located far from stability and therefore can be produced only in a limited amount with low cross sections. In order to perform lifetime experiments under these conditions, we have designed and developed a new plunger device, the TRIple PLunger for EXotic beams (TRIPLEX), which incorporates a target and two degrader foils. This design allows the independent movement of the target and the second degrader with respect to the fixed first degrader, thus simultaneously covering regions of sensitivity for two different lifetimes.

A recoil distance setup using more than the standard target and stopper foil was first proposed in Ref. [2], the so-called differential plunger, where an additional foil, called a retardation (degrader) foil, is placed upstream of the stopper foil at a small distance. This foil only reduces the recoil velocities of nuclei produced and excited in the target but does not stop them completely. Depending on the locations where $\gamma$-ray decays occur along the beam path, three different $\gamma$-ray peaks should appear in the spectra. Those are termed fast, reduced, and stopped (slow) components, each of which corresponds to the $\gamma$-ray 
decay behind the target, behind the degrader, and in the stopper, respectively.

With this configuration, it is possible to determine directly the derivative of the decay function. In practice, this can be approximately done by dividing the reduced-peak yield $I^{r}$ by the time $\Delta t=\Delta x / v$ it takes for reaction products with the speed $v$ to travel over the degrader-stopper distance $\Delta x$. Combined with the amplitude of the decay curve, which can be obtained from the stop-peak yield $I^{s}$, the level lifetime $(\tau)$ can be extracted directly from quantities measured only at one degrader-stopper distance setting as expressed in the following equations:

$$
\begin{aligned}
\tau & =\left|\frac{\exp (-t / \tau)}{\frac{d}{d t}(\exp (-t / \tau))}\right| \\
& \approx \frac{I^{s}}{\frac{1}{\Delta t} I^{r}}=\frac{\Delta x}{v} \frac{I^{s}}{I^{r}}
\end{aligned}
$$

Although this experimental setup in principle has potential advantages, it has never been successfully applied in the past. One reason was the poor separation of the three peaks in low-energy beam experiments with recoil velocities up to only a few percent of the speed of light $(c)$. However, the situation changed when RDDS experiments were applied to rare isotope beams and performed at intermediate beam energies of 100-200 MeV/nucleon [3, 4, 5]. In these experiments, a foil combination of the target and degrader is used (instead of the target and stopper) to identify reaction products coming out of the degrader and final recoiling velocities $(v / c)$ are as high as $20-40 \%$. This triggered the idea of using multiple-foil setups for RDDS experiments and the first attempt was made at GSI measuring $\gamma$-ray decays from ${ }^{36} \mathrm{~K}$ at around $130 \mathrm{MeV} /$ nucleon with three target foils separated at fixed distances [6]. The striking idea of measuring a decay curve at different flight times simultaneously is hampered by the fact that the total statistics of a transition are distributed among different peaks, and normally no real gain in terms of signal-to-noise ratios is obtained from such a setup. Therefore the result was not suited to demonstrate the advantage of a multi-foil plunger setup compared to the standard one. As a result, subsequent RDDS experiments at intermediate energies were focused on the standard twofoil setup and successfully applied to various exotic nuclei $[7,8,9,10,11,12,13]$. 


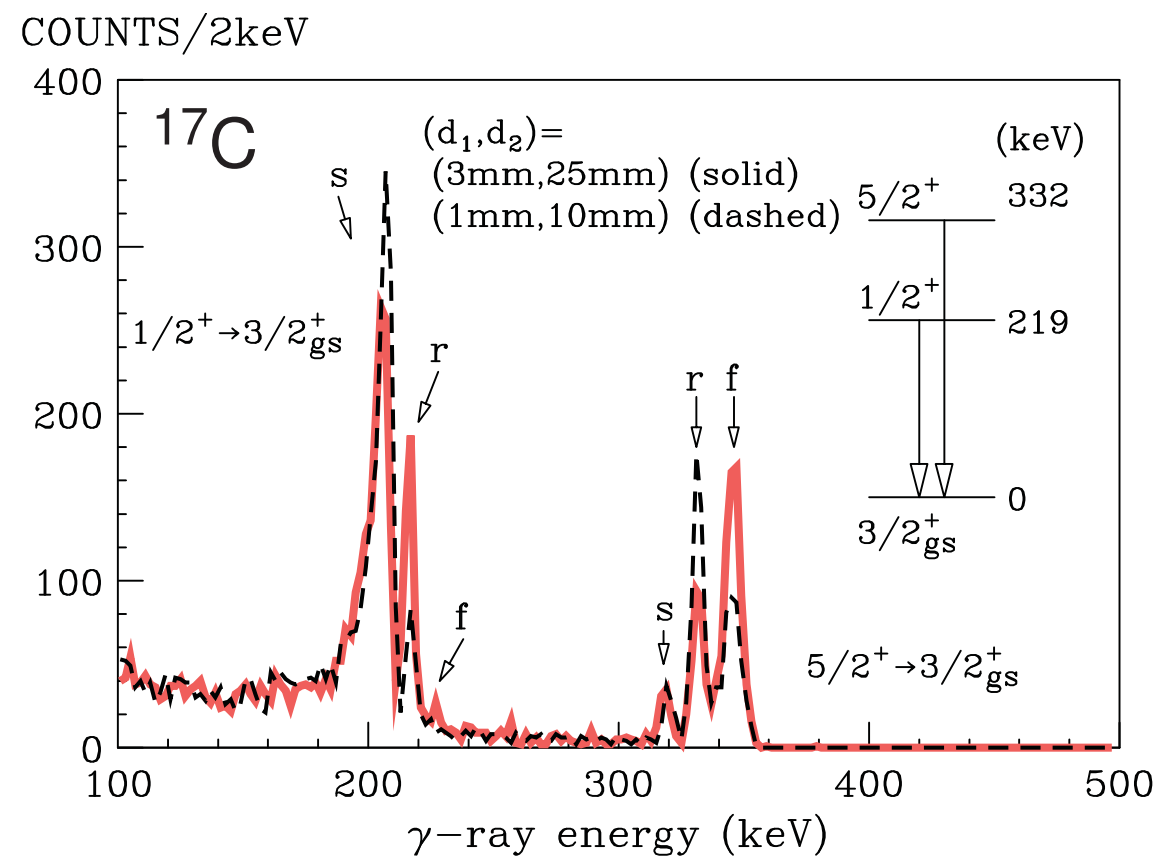

Figure 1: Simulated spectra with a three-foil plunger for ${ }^{17} \mathrm{C}$. The distance $\left(\mathrm{d}_{1}\right)$ between the target and the first degrader and the distance $\left(\mathrm{d}_{2}\right)$ between the first and second degraders are varied from $\left(\mathrm{d}_{1}, \mathrm{~d}_{2}\right)=(3 \mathrm{~mm}, 25 \mathrm{~mm})$ in the solid (red) histogram to $(1 \mathrm{~mm}, 10 \mathrm{~mm})$ in the dashed (black) histogram. Lifetimes of the first $1 / 2^{+}$excited state and the second $5 / 2^{+}$ excited state are assumed to be $\approx 500 \mathrm{ps}$ and $\approx 20 \mathrm{ps}$, respectively. Three different peaks for each transition are denoted as fast (f), reduced (r), and slow (s) components.

Despite the negative results obtained with the three-foil RDDS setup in the past, the idea of taking advantage of the high recoil velocity especially in rare isotope beam experiments was further pursued. Indeed, there are special experimental conditions where a three-foil setup can be advantageous, for instance in cases when lifetimes of two different levels have to be measured with considerably different foil separations. As an example of such cases, lifetime measurements of the neutron-rich nucleus ${ }^{17} \mathrm{C}$ are considered where two independent $\gamma$-ray decays are known for the $1 / 2^{+} \rightarrow 3 / 2_{\text {g.s. }}^{+}$and $5 / 2^{+} \rightarrow 3 / 2_{\text {g.s. }}^{+}$transitions with substantially different lifetimes $\left(\tau \approx 500 \mathrm{ps}\right.$ for $1 / 2^{+}$and $\approx 20 \mathrm{ps}$ for $\left.5 / 2^{+}[14,15]\right)$.

The experimental situation was simulated based on our original code based 
on GEANT4 $[16,17]$ which incorporates modifications to include the three-foil plunger $[18,19]$. The results are shown in Fig. 1 together with the partial level scheme of ${ }^{17} \mathrm{C}$. For the $5 / 2^{+}$state $($at $\sim 330 \mathrm{keV}$ ) with a shorter lifetime, the fast and reduced components provide a useful measure of the lifetime, while the reduced and slow components are much more suited for the lifetime measurement of the longer-lived $1 / 2^{+}$state (at $\sim 220 \mathrm{keV}$ ). The advantage of the multi-foil setup is clearly shown in the simulated results, demonstrating that two different sensitive regions in lifetime measurements can be covered in a single experimental setup. This was our motivation to build a new plunger device where two independent foils (the target and the second degrader) can be used and positioned at different distances with respect to the fixed first degrader.

\section{Design of the three-foil plunger device TRIPLEX}

The TRIPLEX consists of a dedicated plunger vacuum chamber and the inner plunger structure, where the device itself is illustrated in Fig. 2. The design of the device is based on three concentric tubes, where the outer and the inner tubes can be moved precisely along the beam direction with respect to the immobile middle tube, ensuring a parallel motion of the target/degrader foils. Technical specifications are summarized in Table 1. The whole system is comprised of three main parts:

- the support structure

- the bearing unit

- the foil unit.

In the following subsections, details of each component are described.

\subsection{Support structure}

The plunger vacuum chamber, as shown in Fig. 3 (a), hosts the plunger device and is designed to fit into the center of a multi Ge detector array such as SeGA (Segmented Germanium Array) [20] or GRETINA (Gamma-Ray Energy

Tracking In-beam Nuclear Array) [21]. The main part of the chamber consists of 
a 6 inch wide Al tube which is used as a beam pipe and allows positioning of the bare plunger device at an appropriate position inside the $\gamma$-ray spectrometer. The plunger chamber should be quite transparent for $\gamma$ radiation especially in the parts of the solid angle which are covered by the Ge detectors. The actual plunger chamber was designed especially for the specific geometries of the SeGA array as well as GRETINA. Figure 4 shows the plunger setup coupled with GRETINA. The plunger device can be aligned by six special screws attached to the plunger chamber tube (Fig. $3(\mathrm{~b}))$.

It is intended that the downstream flange be permanently fixed to the downstream part of the experimental setup, which is normally attached to a recoil detection system such as a magnetic spectrometer. The plunger chamber also provides several electrical feedthroughs for the cabling of the motors and distance sensors as well as for the electrical connections to the target and degrader foils. The feedthroughs are hosted in two separate chambers which are welded to the plunger chamber tube. The inner plunger structure has to be inserted from the upstream flange of the chamber. In order to do so, the beam tube has to be opened so that at least $50 \mathrm{~cm}$ of free space is available. After having the plunger device inserted and aligned to the beam axis, all cables of specific electrical devices have to be connected to the feedthroughs.

The inner plunger structure is built upon the basic support structure which consists of a support ring and an outer support frame. The support ring, which is connected to the outer support frame, is a basis for all immobile components such as the middle plunger tube, piezoelectrical motors, and connectors. The screws used for the alignment of the plunger device inside the plunger chamber go radially up to the support frame (Fig. 3 (b)). The distance control is performed by two motors directly attached to the support ring, and inductive transducers are used to measure the movements of the foils.

\subsection{Bearing unit}

The bearing unit consists of three stainless steel concentric tubes (Fig. 5) which are separated and held by four sliding bearings attached to the middle 
tube (Fig. 6). This bearing unit is the most crucial part of the plunger device and has to be built with high precision. The spindles of the motors are mechanically connected to the two movable tubes. This connection has to be made such that no radial forces are introduced on the moving tubes. In practice, this is realized by using a short piece of elastic wire (see Fig. 7) to connect the motor and a ring which is clamped to the movable tube. Special care has to be taken to avoid deformation of the tube by the clamped ring and minimize any force onto the slide bearings.

\subsection{Foil unit}

Opposite the clamp ring, a brass ring is fixed to the end of each tube which allows the foil unit to be mounted. The foil unit consists of three thin $\mathrm{Al}$ frames which are attached to the brass rings of the tubes and, at the other end, have a provision to mount the target and degrader foils. The foils are glued to mounting cones, which are in turn connected to the Al frames by three screws. The cones are electrically insulated by dedicated boron nitride pieces to enable a capacitance measurement between the foils which is required to evaluate the foil separation. Hard springs are introduced for the connection so that the screws can press and release to align the three foils parallel to each other and perpendicular to the beam axis. Since the beam has to pass through the inner tube to reach the target, the width of the target and degrader foils was chosen to be $\approx 5 \mathrm{~cm}$ (Table 1 ) which is essential especially for measurements with fast rare isotope beams where the beam spot size can be as large as a few centimeters.

To mount the three target or degrader foils, three independent cones are necessary. As shown in Fig. 8, two cones are positioned face to face, while the third cone needs to enter into the second cone which requires different dimensions for the foils. Therefore, the cone for the second degrader is prepared in a special shape (Fig. 8) and the second degrader in a circular shape has to fit into the inner diameter of the first degrader cone. The alignment of the foils is done by adjusting the screws which mount the cones and the optimal setting can be achieved by maximizing the capacitance between foils. However, the 
three-foil structure of the TRIPLEX requires a two-step procedure for the foil alignment: first, the target is aligned with respect to the fixed degrader and next, the second degrader is aligned with respect to the aligned target with the first degrader removed. By inserting the first degrader again in its fixed place, the capacitance measurement between the target and first degrader can be repeated and, if the capacitance is comparable to the previous measurement, the three foils are aligned.

\section{Control software}

The Plunger Feedback Control software for Linux (PFCL) was initially developed to control the Cologne plunger [1]. The programs were also adopted to work with other plunger devices, such as DPUNS [22]. From a software point-ofview, the TRIPLEX can be considered to some extent as two different plunger devices accommodated within one housing. Existing frameworks are therefore expanded into a modular concept so that multiple devices can be handled in parallel to fulfill comparable tasks.

\subsection{Controlled components}

The software package is written in LabVIEW and is able to control both active (position control elements such as motors and piezoelectric elements) and passive elements (distance measurement). In the TRIPLEX, both the target and second degrader are aligned with respect to the first degrader at a fixed position between these two foils. The N-381 NEXACT linear actuator manufactured by Physik Instrumente (PI) [23] is employed as a motor to handle well-defined linear displacements of the target and second degrader. The linear actuator is equipped with a linear encoder which provides the distance information with sub- $\mu \mathrm{m}$ resolution. The inductive-working micrometer probe GTL21 manufactured by TESA group [24] is used to precisely measure relative changes in the foil separations with sub- $\mu \mathrm{m}$ resolution. These measurements done by the encoder and micrometer offer a relative distance information (Table I). In 
low-energy beam experiments $[22,25,26]$, thermal effects may affect the foil separations, if large beam powers are dissipated in the foils. In this case, a feedback function based on the capacitance measurement is mandatory to compensate small changes in the foil separation (usually in the $\mu \mathrm{m}$ range) and keep the separation constant during measurements. For a potential use in reaccelerated rare isotope beam experiments as envisioned by other plunger systems for different facilities [25, 26], the TRIPLEX device is equipped with a feedback function which can be performed either by using the motor or an additional piezoelectric element. Since the capacitance feedback needs to translate the measured capacitance into the position information, a calibration of the relative foil separation is necessary prior to experiment.

\subsection{Usage of control software}

The current control program PFCL is object-orientated and follows a modular concept so that several hardware components can be grouped into classes according to their tasks. Classes for the following tasks are currently prepared: Major $(>10 \mu \mathrm{m})$ linear displacements (by motors), minor (on the order of $\mu \mathrm{m}$ ) linear displacements (by piezoelectric elements or by the motors), distance measurements, and a triggered voltage readout. The circuit diagram between the TRIPLEX device and the control PC is illustrated in Fig. 9. The modular software concept allows handling of multiple devices of the same class, while an external configuration file is used to specify the configuration for each experiment. For practical usage of the current software, several graphical user interfaces (GUI) are prepared to control the plunger in an intuitive way. The Micrometer GUI (Fig. 10 (a)) is used to read out the relative position information provided by the micrometer probe. The Motor GUI (Fig. 10 (b)) is used to drive the motor with various velocity settings $(\mathrm{v} \approx 0.5 \mu \mathrm{m} / \mathrm{s}-0.2 \mathrm{~mm} / \mathrm{s})$ and display the distance information from the embedded linear encoder. Finally, if a pulser signal is injected to the fixed degrader, induced signals can be measured from the target and the second degrader (Fig. 9). The induced signals go through NIM modules as shown in Fig. 9 and their amplitudes can be measured 
with the multichannel analyzer using the MCA GUI (Fig. 10 (c)). A linear gate and stretcher is used to accommodate a possible time jitter between the pulser signal and the start timing of the ADC conversion and measure a proper induced signal by MCA. The distance calibration is made by recording the amplitudes of the induced signals for different separations, and the feedback, if needed during experiments, is applied based on the measured pulse heights of the induced signals. For a practical operation, the position of the electrical contact between foils is defined as a zero distance setting, while it could include a finite offset on the order of $10 \mu \mathrm{m}$ (Table I). To determine the absolute foil separation, it is necessary to know this offset, which is normally evaluated based on the capacitance measurement [27] by comparing the distance calibration data to an analytical form of the parallel-plate capacitance.

\section{Simulations and experimental results}

Based on its unique design, the TRIPLEX allows advanced approaches in excited-state lifetime measurements with fast rare isotope beams. In this section, two new applications of the recoil distance Doppler-shift technique are discussed. Since the TRIPLEX setup allows the independent motion of the target and second degrader foils, two quantities are defined for the separation $\left(\mathrm{d}_{1}\right)$ between the target and the first degrader and the separation $\left(\mathrm{d}_{2}\right)$ between the first and second degraders. The dynamic range for the foil separation is between $0 \mathrm{~mm}$ and $25 \mathrm{~mm}$ for both $\mathrm{d}_{1}$ and $\mathrm{d}_{2}$ (Table 1). Firstly, the differential recoil distance method is discussed in detail, which is based on the measurement of the differential and integral values of the decay curve using the reduced and slow peaks, respectively [2]. Secondly, the double recoil distance method is described, where the distance setting with small $\mathrm{d}_{1}$ and large $\mathrm{d}_{2}$ separations enables a simultaneous measurement of two different lifetimes using a single experimental setup. Characteristics and sensitivities of the above two methods are discussed based on existing GEANT4 simulations $[17,18]$ which have already been used in recent lifetime studies performed at the National Superconducting Cyclotron 
Laboratory (NSCL) with GRETINA [13, 15, 19].

In the present simulation, the following conditions are considered. The inelastic scattering of an incoming beam ${ }^{A} Z$ with $A=60$ and $Z=32$ at $90 \mathrm{MeV} /$ nucleon on a Be target is employed in inverse kinematics to populate excited states of interest. It is assumed that the reaction populates two excited states at $300 \mathrm{keV}$ and $800 \mathrm{keV}$ in the differential recoil distance method (at $300 \mathrm{keV}$ and $500 \mathrm{keV}$ in the double recoil distance method) which have different lifetimes $\tau_{1}$ and $\tau_{2}$, respectively. The lifetime values are changed to study features of each method. The foil configuration of the TRIPLEX is a 1-mm-thick Be target, a 0.1-mm-thick Ta degrader, and a 0.1-mm-thick Ta degrader. In general, nuclear reactions to populate the excited states of interest can occur both in the target and the degraders. However, a proper choice of the degrader materials [4] as well as an additional momentum selection of reaction products by means of a magnetic spectrometer helps to reduce or eliminate the background contributions due to the reactions in the degraders. In the present setting, the areal density is about twenty times larger for the Be target than that of the Ta degrader so that the excited-state population is dominated by reactions in the target. In actual experiments, background contributions due to the reactions in the degraders are evaluated by measuring the yield ratios among the three different $\gamma$-ray peaks at a largest possible foil separation. Typical background levels are around $20-30 \%$ [7, 19] with respect to the target reaction yields. Other experimental parameters such as the spot size and emittance of incoming and outgoing beams are assumed to be the same as observed in recent measurements at NSCL $[13,15,19]$, because the impacts of these parameters on present results are rather marginal.

\subsection{Differential recoil distance measurement}

As performed in recent works with the TRIPLEX [13, 15, 19], lifetimes are determined through the least-squares analysis by comparing data to detailed simulations [17]. The advantage of this approach is that the simulation can take into account various experimental details such as feeding contributions, finite 
target/degrader effects, and $\gamma$-ray decays occurring in the foils, which can not be fully represented by the simple analytical approach based on the reduced/slow yield ratio as shown in Eq. (2). However, as discussed below, an estimate based on the differential equation provides a transparent interpretation of the data, which is useful at the initial stage of the analysis and ensures the reliability of final experimental results from the differential recoil distance method.

The systematic uncertainties in recoil distance lifetime measurements are mainly due to unknown feeding contributions from higher-lying states. If such a transition is known or observed in experiments, the feeding effects can be carefully taken into account in the analysis. However, if the origin of the feeding and its effective lifetime are unknown, the treatment of the feeding contributions becomes very difficult unless the experimental statistics are large enough to apply the gamma-gamma coincidence to eliminate the influence of the feeding $[28,29]$. To realize a transparent approach in lifetime measurements, the Differential Decay-Curve Method (DDCM) [2] has been applied so far (examples can be found in Refs. $[1,30])$, in which a number of data points are taken with various distance settings and a phenomenological decay curve is determined. The derivative of the curve is obtained analytically and the original data points for the decay curve are divided by its derivative value, yielding the mean lifetime as expressed in Eq. (1). Although this technique offers a powerful tool to obtain an accurate result, it requires taking data with various foil separations, which is often not possible in experiments with rare isotope beams. Therefore, the new TRIPLEX device has been developed such that a single distance measurement allows the determination of both the decay integral $\exp (-t / \tau)$ and its derivative $\frac{d}{d t} \exp (-t / \tau)$ from the measured slow and reduced peak yields, respectively, and hence achieves the differential recoil distance measurement.

Figure 11 shows simulated spectra for the differential recoil distance measurement. The distance setting of $\left(\mathrm{d}_{1}, \mathrm{~d}_{2}\right)=(2 \mathrm{~mm}, 2 \mathrm{~mm})$ is considered to show a specific example of actual applications. Compared to the double recoil distance method depicted in Fig. 1, the major difference is given by the short separation for $\mathrm{d}_{2}$. In the present case, a cascade decay is considered where the 
reaction populates exclusively the second excited state at $800 \mathrm{keV}$ and the first excited state at $300 \mathrm{keV}$ can be populated only through the feeding from the second excited state. In Fig. 11 (a), a mean lifetime of 20 ps is considered for the first excited state, whereas a very short lifetime of $0.1 \mathrm{ps}$ is taken for the second excited state to minimize feeding effects. In such a case, $\gamma$ decays could occur inside of the thick target. Therefore, the spectral shape of the fast component reflects the large energy loss of the projectile $(Z=32)$ through the target, which results in the broadened or asymmetric peak. A smaller separation for $d_{2}$ is ideal for getting the differential value, however $\mathrm{d}_{2}$ needs to be sufficiently large to ensure statistical significance for the reduced peak. By following Eq. (2), the lifetime information can be obtained from the ratio between the reduced $I^{r}$ and slow $I^{s}$ peak yields. From Gaussian fits to the spectrum in Fig. 11 (a), the $I^{s} / I^{r}$ ratio of $\sim 0.6$ is obtained for the $300-\mathrm{keV}$ state, which yields the mean lifetime of $\sim 12$ ps after multiplying the time of flight $\Delta t$ of $\approx 20$ ps (with $v / c \approx 0.3$ ) over the second separation of $2 \mathrm{~mm}$. The result is much shorter than the assumed lifetime of $20 \mathrm{ps}$, and the difference is ascribed to the approximation to calculate the first derivative of the decay curve based on the reduced component. The analytical form of the derivative is given (if a normalization factor is neglected):

$$
\left.\frac{d}{d t}(\exp (-t / \tau))\right|_{t=t_{2}}=-\frac{1}{\tau}\left(\exp \left(-t_{2} / \tau\right)\right) .
$$

On the other hand, the derivative can be approximately obtained from the measured spectrum as:

$$
-\frac{1}{\Delta t} I^{r}=\left(\exp \left(-t_{2} / \tau\right)-\exp \left(-t_{1} / \tau\right)\right) /\left(t_{2}-t_{1}\right)
$$

where $t_{1}$ and $t_{2}$ specify times when recoiling nuclei leave the first degrader $\left(t_{1}\right)$ and enter the second degrader $\left(t_{2}\right)$, respectively. The difference between the above two quantities is not negligible for the finite foil separation and amounts to as large as a factor of 1.7 for the present case where the time of flight $\Delta t(=$ $t_{2}-t_{1}=20 \mathrm{ps}$ ) is comparable to the lifetime $\tau$. However, the ratio of Eq. (4) to Eq. (3) defines a correction factor $C(\tau, \Delta t)$ :

$$
C(\tau, \Delta t)=\frac{\exp (\Delta t / \tau)-1}{\Delta t / \tau}
$$


and a correct lifetime can be deduced as follows:

$$
\tau^{c o r}=\Delta t \frac{I^{s}}{I^{r}} C(\tau, \Delta t)
$$

In actual cases, the lifetime information is unknown, while it is required to calculate $C(\tau, \Delta t)$. Therefore, an iteration process is taken to use Eq. (6). Initially, an estimated lifetime based on Eq. (2) can be used for $C(\tau, \Delta t)$ to obtain a corrected lifetime. This result gives, in the next turn, a new correction factor and hence a revised lifetime. After several iterations (typically more than 10), the results converge to an accurate value. As shown in Eq. (5), the correction factor depends only on the ratio between the time-of-flight $\Delta t$ over the second separation $\mathrm{d}_{2}$ and the lifetime $\tau$ and it approaches unity when $\Delta t / \tau \ll 1$. In experiments, a finite value of $\Delta t / \tau$ can be chosen so that sufficient yields are obtained for both the reduced and slow peaks, whereas $\Delta t / \tau$ should be smaller than $\approx 1.5$ to have convergence through Eq. (6). In the present case, the correction factor is about 1.7 for $\Delta t / \tau=1$ and the final result can be calculated to about $20 \mathrm{ps}(=12 \mathrm{ps} \times 1.7)$, which agrees very well with the original lifetime assumed in the simulation.

To study impacts of feeding contributions, in Fig. 11 (b), the lifetime for the second excited state is changed to $10 \mathrm{ps}$. The feeding effects in the cascade decay can be easily seen in the modified spectral shape of the $300-\mathrm{keV}$ peak, particularly in its fast component. Notably, the reduced/slow peak ratio remains unchanged compared to Fig. 11 (a), showing the ability of the present differential method to control and reduce the feeding effects, particularly in this specific example. The $I^{s} / I^{r}$ ratio of $\sim 0.7$ estimated in this case corresponds to the initial estimate of the lifetime of $14 \mathrm{ps,} \mathrm{which} \mathrm{yields} \mathrm{the} \mathrm{final} \mathrm{result} \mathrm{of} 23 \mathrm{ps}$ after corrections based on Eq. (6). It should be noted that the final result is still in reasonable agreement with the original lifetime of 20 ps even with the significant feeding from the second excited state as is evident in the altered fast/reduced ratio in Fig.11 (b). 


\subsection{Double recoil distance measurement}

As briefly discussed in the introduction, the TRIPLEX measurement with two movable foils can cover two different sensitive regions of lifetimes simultaneously. At the typical recoil velocity of $v / c \approx 0.3$ at NSCL, beams travel a distance of about $1 \mathrm{~mm}$ in $10 \mathrm{ps}$. Therefore, two different lifetimes in the ranges of $10 \mathrm{ps}$ and $100 \mathrm{ps}$ can be covered by setting the first separation $\mathrm{d}_{1} \approx 1 \mathrm{~mm}$ and the second separation $\mathrm{d}_{2} \approx 10 \mathrm{~mm}$ in the TRIPLEX. In this application, lifetimes are determined by varying both separations around the sensitive regions as shown in Fig. 1.

Figure 12 shows simulated spectra for the double recoil distance measurements. Lifetimes assumed in Fig. 12 (a) (and also in dashed lines of Fig. 12 (b) and (c)) are $\tau_{1}=100 \mathrm{ps}$ for the first excited state at $300 \mathrm{keV}$ and $\tau_{2}=10$ ps for the second excited state at $500 \mathrm{keV}$. Both of the excited states decay directly to the ground state. The same population cross section is assumed for the two states. To depict a feature of this method, in Fig. 12 (b) and (c), lifetime values are changed from (50 ps, 5 ps) (thick red), to (100 ps, 10 ps) (black dashed), and to (150 ps, $15 \mathrm{ps)} \mathrm{(thin} \mathrm{blue)} \mathrm{for} \mathrm{the} \mathrm{first} \mathrm{and} \mathrm{second} \mathrm{excited} \mathrm{states,} \mathrm{respectively.}$ Effects due to the lifetime are evident in the yield distribution among three different Doppler-shift components, which are shifted toward the reduced or slow components for longer lifetimes. More importantly, the three $\gamma$-ray peaks have different sensitivities: for a longer lifetime, the reduced/slow peak ratio provides a useful means for lifetime measurements (Fig. 12 (b)), while the fast/reduced peak ratio is more sensitive to the shorter lifetime as indicated in Fig. 12 (c), demonstrating the important feature of the three-foil configuration for multiple lifetime measurements. If a broadened or asymmetric spectral shape is evident for the fast component as shown in Fig. 12 (c), it also provides an additional means to determine lifetimes in the $1-5$ ps range [19]. The advantage of the three-foil approach is also demonstrated by comparing Fig. 12 (a) and Fig. 13, where the latter shows simulation results for the traditional two-foil plunger measurements. The experimental conditions are the same except for the absence of the second degrader in Fig. 13. The three-foil setup can cover a wider 
range of lifetimes and provide two distance data at the same time, while the two-foil setup requires two independent measurements with different distances to get equivalent information.

As an example of experimental results, the $\gamma$-ray spectrum observed for ${ }^{17} \mathrm{C}$ is shown in Fig. 14 and compared to the simulation. The experiment was performed at the Coupled Cyclotron Facility at NSCL using a $74 \mathrm{MeV} /$ nucleon ${ }^{18} \mathrm{C}$ secondary beam onto a 2 -mm-thick ${ }^{9}$ Be target followed by 1 -mm-thick Ta degrader and 0.57-mm-thick Ta degrader. De-excitation $\gamma$-rays were measured by GRETINA [21] in coincidence with outgoing ${ }^{17} \mathrm{C}$ particles identified by the S800 spectrograph [31]. Only forward-angle detectors are used in the lifetime analysis and simulation with a further angular cutoff $\left(\theta_{l a b}<40^{\circ}\right)$ applied. Four different recoil distance measurements were performed with various distance settings, and the result from one setting $\left(\mathrm{d}_{1}=3 \mathrm{~mm}, \mathrm{~d}_{2}=22.8 \mathrm{~mm}\right)$ is shown in the spectrum. The detailed description of the experiment and discussion of obtained results will be published elsewhere [15].

As shown in Fig. 14, the well-separated peak structure is evident for both the $1 / 2^{+} \rightarrow 3 / 2_{\text {g.s. }}^{+}$transition at $220 \mathrm{keV}$ and the $5 / 2^{+} \rightarrow 3 / 2_{\text {g.s. }}^{+}$transition at $330 \mathrm{keV}$. Due to the sizable degrader reaction contributions in this specific experiment, additional yields are present for the reduced and slow components. Therefore, the measured yield distribution among the three peaks is slightly different from that expected from the simulation (the solid curve in Fig. 1). Nevertheless, it is clearly shown that the TRIPLEX setup can cover two independent lifetime regions in a single experiment. Results obtained [15] are consistent with the previous work [14]. This demonstrates the feasibility and usefulness of the double recoil distance method for efficient lifetime measurements using rare isotope beams.

\section{Summary}

In summary, the three-foil plunger device TRIPLEX has been developed to enable advanced recoil distance techniques in excited-state lifetime measure- 
ments with fast rare isotope beams. The unique design and control software allow the independent motion of the two foils and also facilitate the stable operation of this new device during experiments. As new techniques for which the TRIPLEX is ideally suited, the differential recoil distance method and the double recoil distance method are discussed in detail based on the simulation results. The TRIPLEX device has already been used in several lifetime experiments at NSCL in the standard two-foil configuration $[12,13,32]$ and the new three-foil configuration $[15,19]$. The result presented for the neutron-rich ${ }^{17} \mathrm{C}$ isotope demonstrates the promising performance of the new lifetime measurement techniques realized by the three-foil plunger.

\section{Acknowledgement}

The authors would like to thank the mechanical workshop at IKP, University of Cologne, for their excellent work. This work is supported by the National Science Foundation (NSF) under PHY-1102511, by the Department of Energy (DOE) National Nuclear Security Administration under award number DENA0000979, and partly by DFG under DE1516/3-1. GRETINA was funded by the US DOE - Office of Science. Operation of the array at NSCL is supported by NSF under Cooperative Agreement PHY-1102511 (NSCL) and DOE under grant DE-AC02-05CH11231 (LBNL).

\section{References}

[1] A. Dewald, et al., Prog. Part. Nucl. Phys. 67, 786 (2012).

[2] A. Dewald, et al., Z. Phys. A 334, 163 (1989).

[3] K. Starosta et al., Phys. Rev. Lett. 99, 042503 (2007).

[4] A. Chester et al., Nucl. Instr. Meth. A 562, 230 (2006).

[5] A. Dewald, et al., Phys. Rev. C 78, 051302(R) (2008).

[6] P. Bednarczyk et al., Acta. Physica Polonica B 41, 505 (2010). 
[7] W. Rother et al., Phys. Rev. Lett. 106, 022502 (2011).

[8] M. Petri et al., Phys. Rev. Lett. 107, 102501 (2011).

[9] M. Niikura et al., Phys. Rev. C 85, 054321 (2012).

[10] M. Petri et al., Phys. Rev. C 86, 044329 (2012).

[11] P. Voss et al., Phys. Rev. C 86, 011303(R) (2012).

[12] A. Nichols et al., Phys. Lett. B 733, 52 (2014).

[13] K. Whitmore et al., Phys. Lett. C 91, 041303(R) (2015).

[14] D. Suzuki et al., Phys. Lett. B 666, 222 (2008).

[15] D. Smalley et al., submitted.

[16] S. Agostinelli et al., Nucl. Instr. Meth. A 506, 250 (2003).

[17] P. Adrich et al., Nucl. Instr. Meth. A 598, 454 (2009).

[18] A. Lemasson, et al., Phys. Rev. C 85, 041303(R) (2012).

[19] H. Iwasaki et al., Phys. Rev. Lett. 112, 142502 (2014).

[20] W. Mueller et al., Nucl. Instr. Meth. A 466, 492 (2001).

[21] S. Paschalis et al., Nucl. Instr. Meth. A 709, 44 (2013).

[22] M.J. Taylor et al., Nucl. Instr. Meth. A 707, 143 (2013).

[23] http://www.physikinstrumente.com/

[24] http://www.tesagroup.com/

461 [25] J. Ljungvall et al., Nucl. Instr. Meth. A 679, 61 (2012).

${ }_{62}^{62}$ [26] P. Voss et al., Nucl. Instr. Meth. A 746, 87 (2014).

63 [27] T.K. Alexander, A. Bell, Nucl. Instr. Meth. 81, 22 (1970).

[28] G. Böhm et al., Nucl. Instr. Meth. A 329, 248 (1993). 
[29] P. Petkov et al., Nucl. Instr. Meth. A 457, 527 (2001).

466 [30] A. Görgen, et al., Eur. Phys. J A 26, 153 (2005).

${ }_{467}^{631]}$ D. Bazin et al., Nucl. Instr. Meth. B 204, 629 (2003).

${ }_{468}$ [32] T. Braunroth et al., accepted for publication in Phys. Rev. C. 

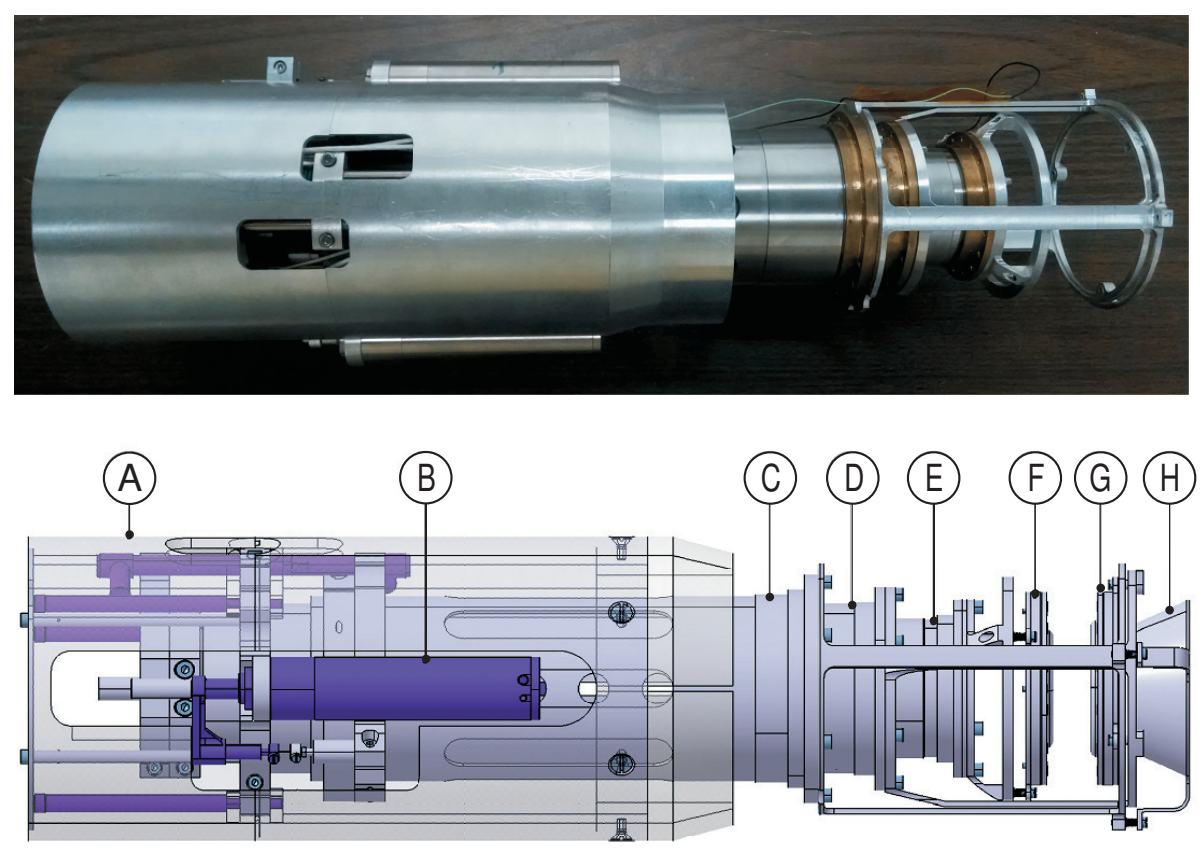

Figure 2: The TRIPLEX device (top : photograph, bottom : drawing). The components are labeled in the bottom panel as follows: (A) The outer casing (support frame) of the TRIPLEX. (B) One of the piezoelectric motors (linear actuators) for positioning the target foil. (C) The outermost movable tube, to which the second degrader is attached. (D) The central, immobile tube to which the first degrader is attached. (E) The innermost movable tube to which the target is attached. (F) The target, positioned by the movable tube E. (G) The first degrader, held at a fixed position. (H) The second degrader frame, on which the second degrader (obscured by the first degrader) is mounted. In the top photograph, the target and degraders are removed to show a frame structure of the device. 
Table 1: A summary of technical details of the TRIPLEX device. The origin of the movable range is at the fixed first degrader and the axis is defined toward the direction of the beam. For the geometry setting labeled with (a), typical values in experiments are listed.

\begin{tabular}{l|c}
\hline \hline Movable range for the target & $-25-0 \mathrm{~mm}$ \\
\hline Movable range for the second degrader & $0-25 \mathrm{~mm}$ \\
\hline Clearance in the beam path & $\geq \varnothing 45 \mathrm{~mm}$ \\
\hline Dimension of the target/the first degrader & $50 \times 50 \mathrm{~mm}^{2}$ \\
\hline Dimension of the second degrader & $\varnothing 47.5 \mathrm{~mm}$ \\
\hline Length of the device & $\sim 50 \mathrm{~cm}^{(a)}$ \\
\hline $\begin{array}{l}\text { Precision of } \\
\text { the relative distance measurement }\end{array}$ & $1 \mu \mathrm{m}$ \\
\hline Offset for the foil separation & $\lesssim 30 \mu \mathrm{m}^{(a)}$ \\
\hline \hline
\end{tabular}


(a)

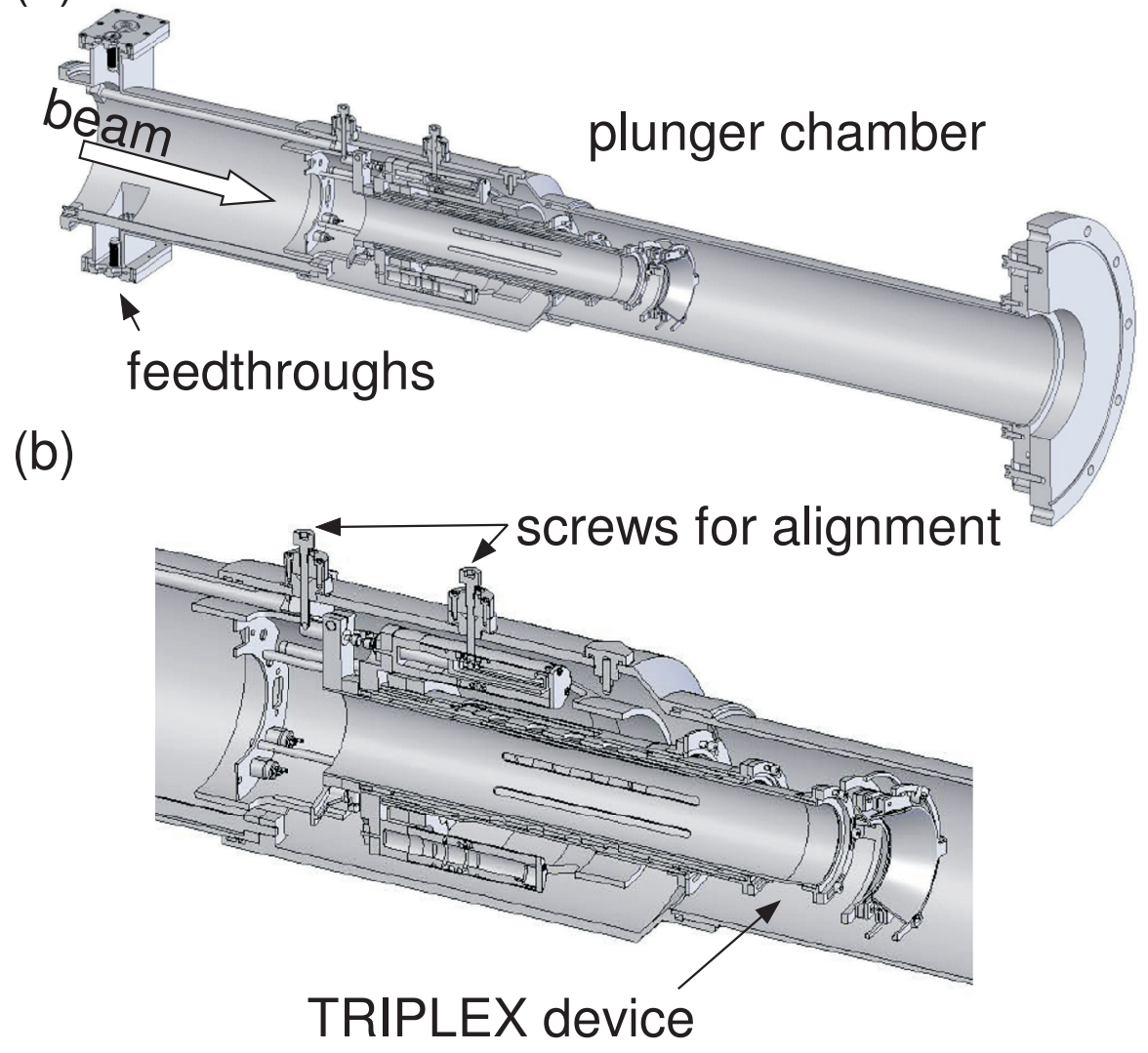

Figure 3: The plunger chamber holding the TRIPLEX device inside is shown in (a), together with a close-up view (b). 


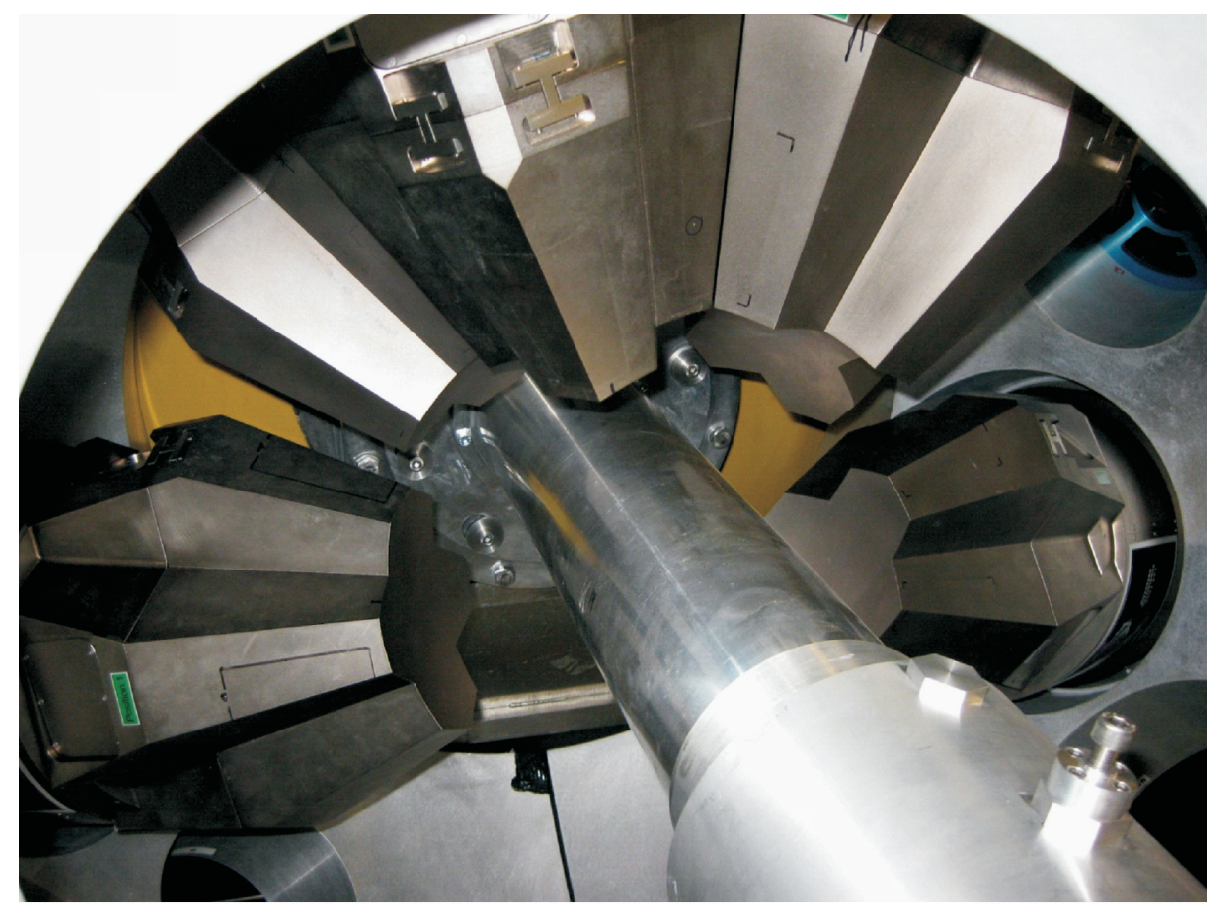

Figure 4: The TRIPLEX chamber installed with GRETINA [21].

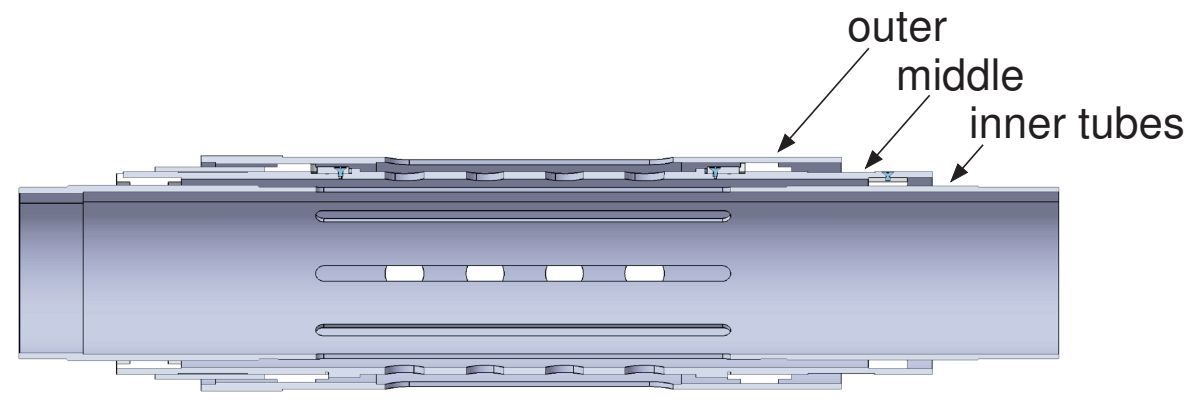

Figure 5: The structure of the three tubes connected to each plunger target/degrader frame is shown. The outer and inner tubes are movable with respect to the stationary middle tube. See also Fig. 8 for a close-up view. 


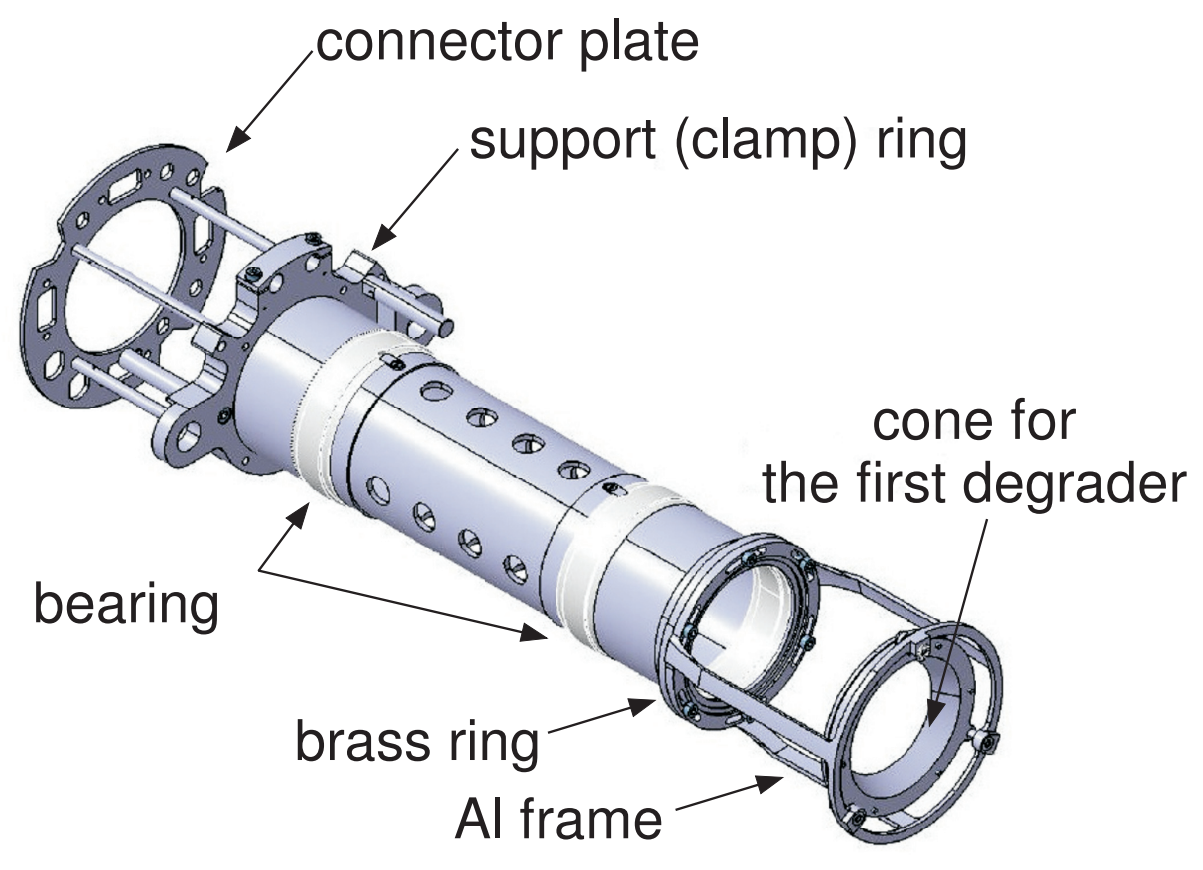

Figure 6: A schematic view of the middle tube which is clamped to the support ring. Four sliding bearings (light grey) are placed on both the inner and outer sides of the middle tube.

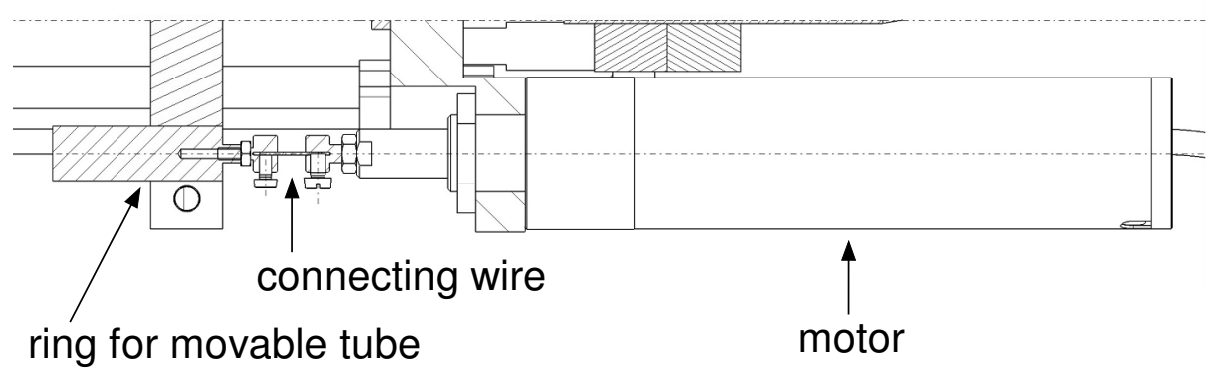

Figure 7: An example of the connection between the movable tube and the head of motor through a thin wire. For the TRIPLEX, the piezoelectric element is embedded in the ring for the movable tube and connected with the motor through the wire. 


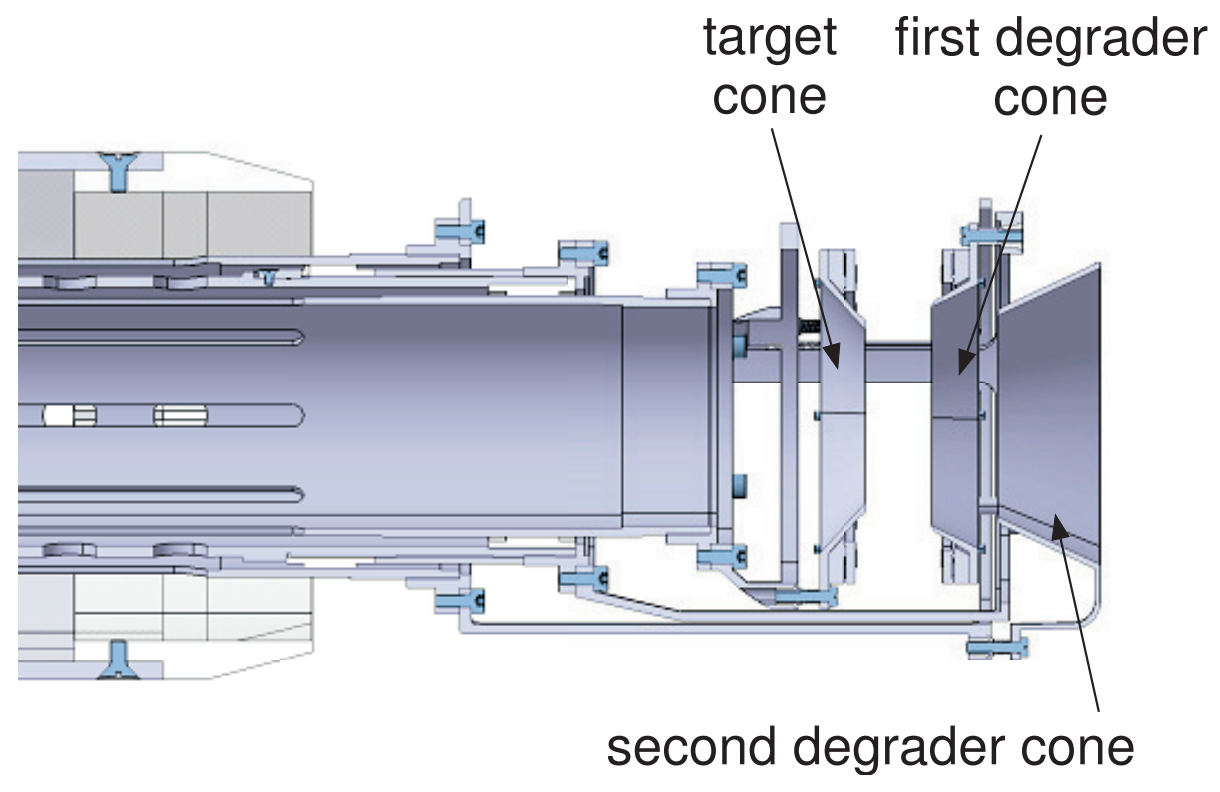

Figure 8: The close-up view of the TRIPLEX device showing three cones to mount the target and degrader foils. 


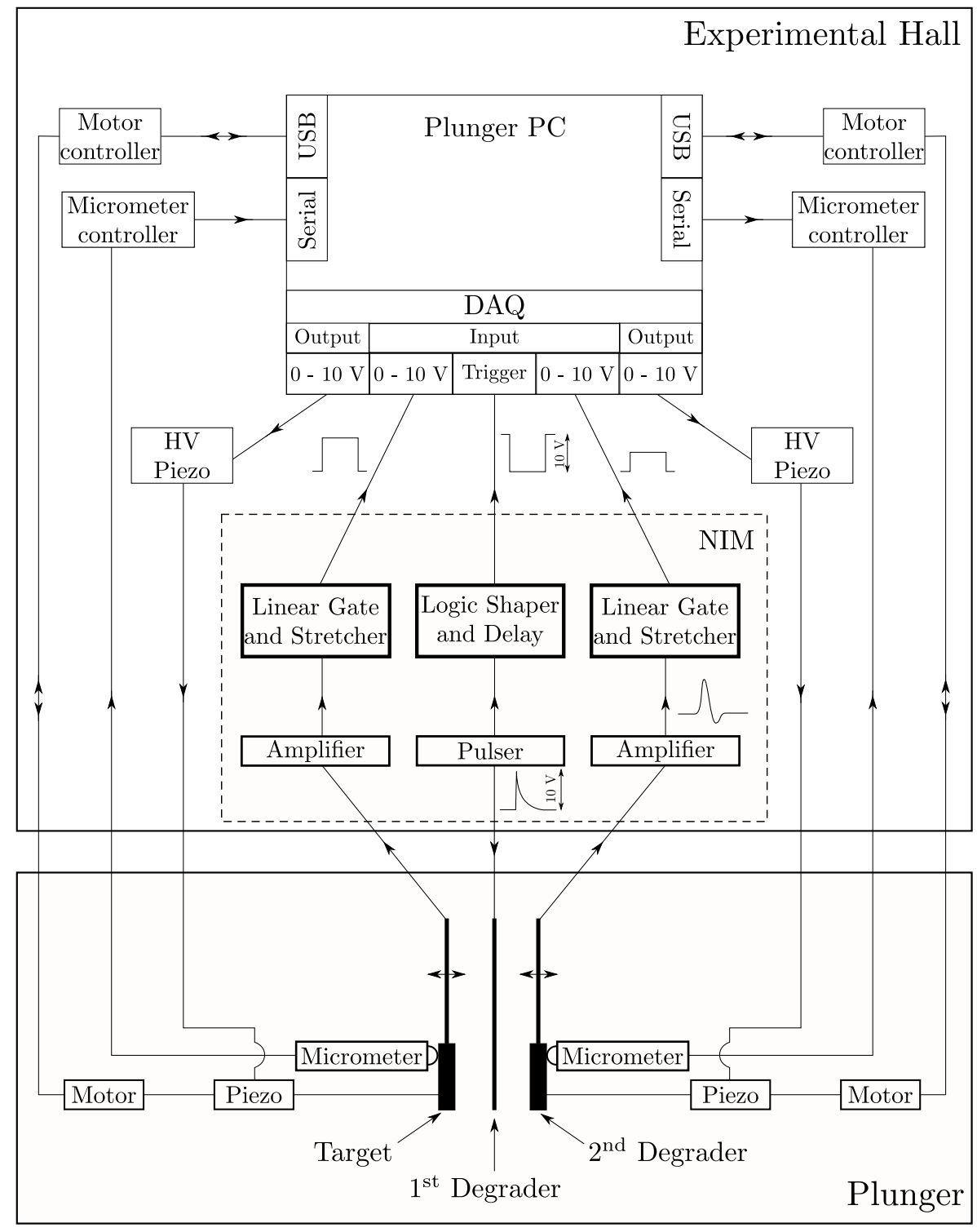

Figure 9: A schematic diagram of the electronic modules to control the TRIPLEX. 


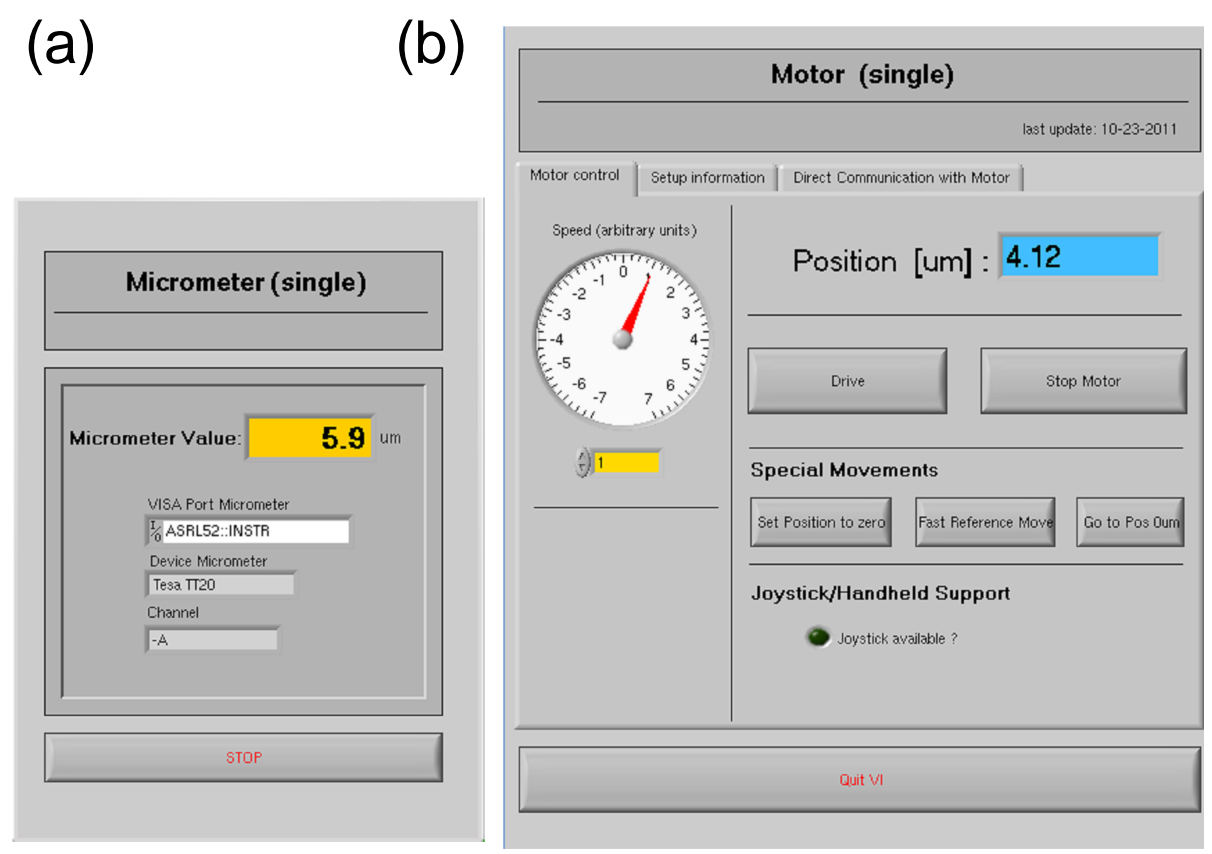

(c)

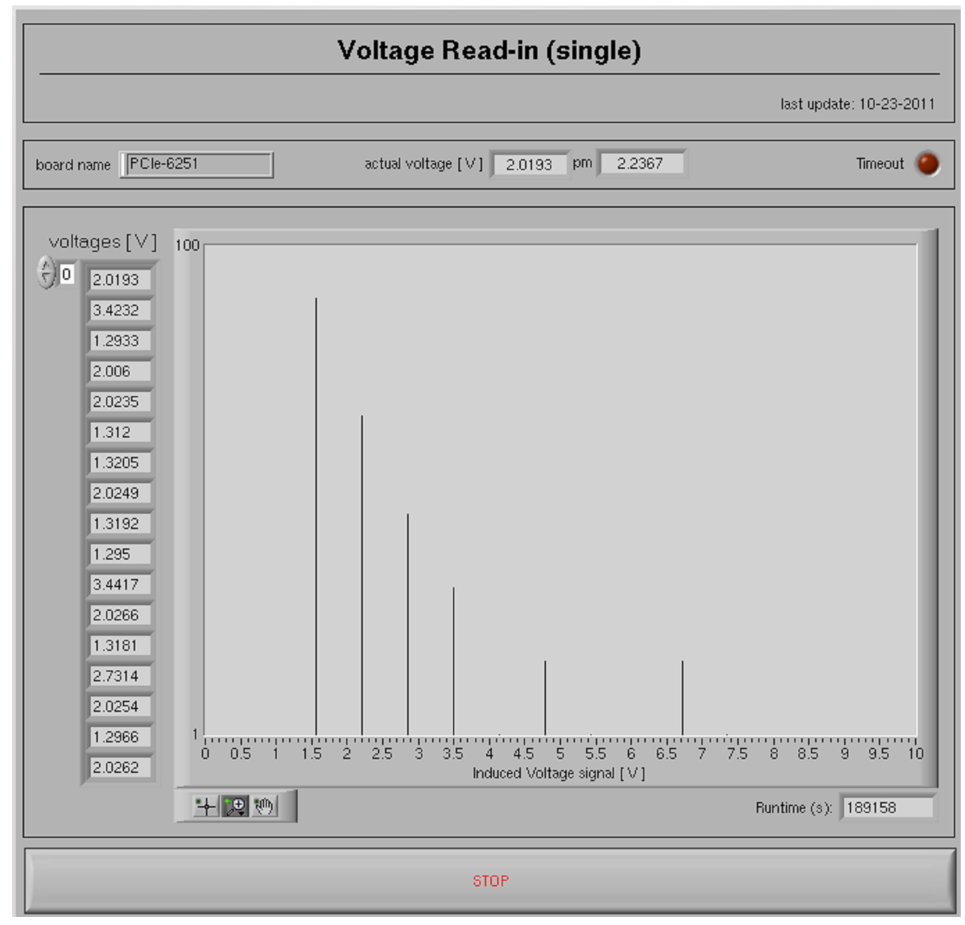

Figure 10: Examples of graphical interfaces (GUI) for user control: (a) position measurement based on the micrometer (Micrometer GUI), (b) control and position monitoring of the motor (Motor GUI), and (c) induced signal measurement with the multi-channel analyzer (MCA GUI). Graphical interface windows to contro $2 \not$ single plunger are shown in the figure, while the same control panel is duplicated in each device window when two motors are operated for the TRIPLEX. 


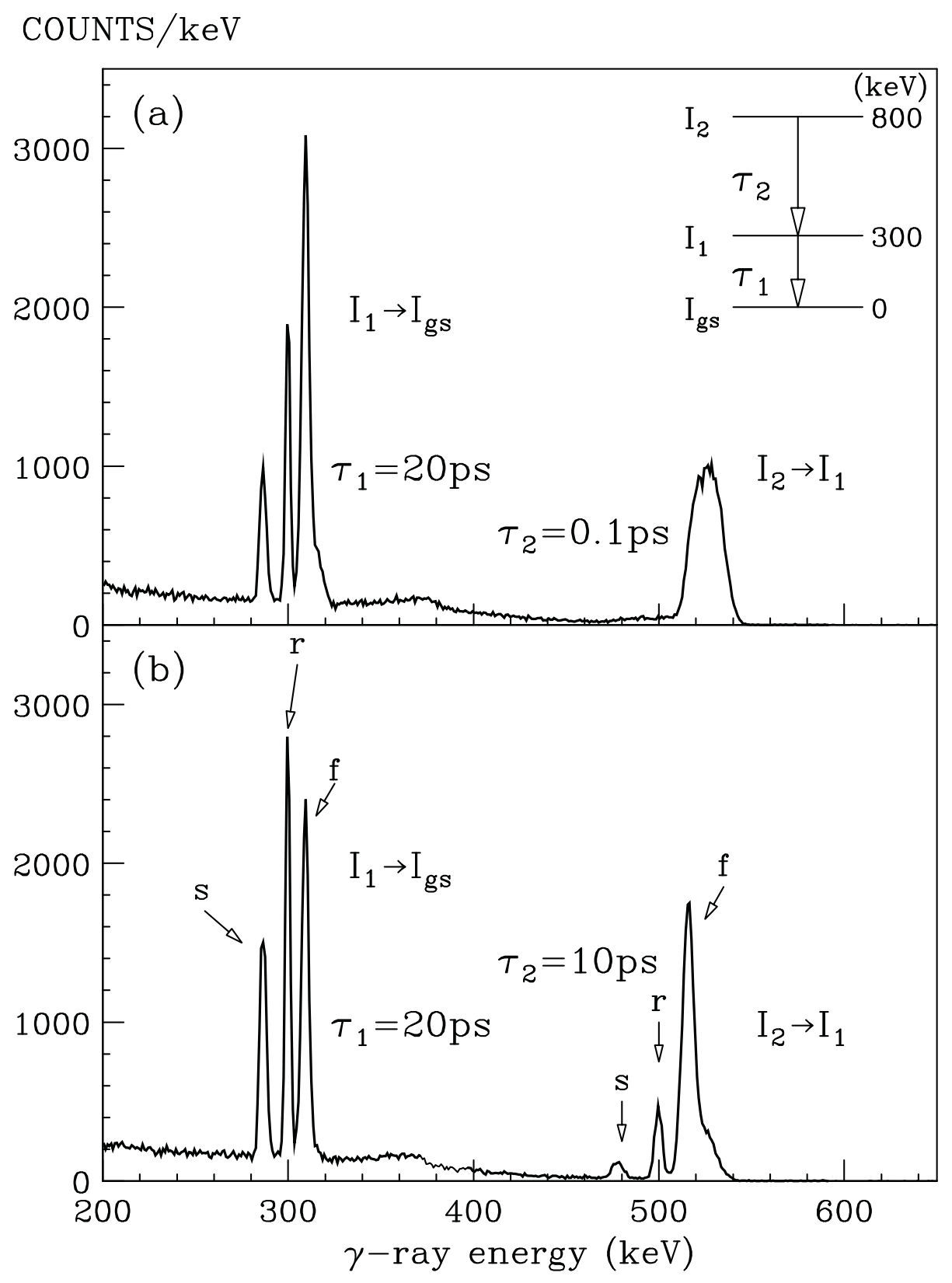

Figure 11: Simulated spectra are shown for the differential recoil distance measurements with the distance setting $d_{1}=2 \mathrm{~mm}$ and $d_{2}=2 \mathrm{~mm}$. The mean lifetimes for the first and second excited states $\left(\tau_{1}, \tau_{2}\right)$ are assumed to $(20 \mathrm{ps}, 0.1 \mathrm{ps})$ in (a) and (20 ps, $\left.10 \mathrm{ps}\right)$ in (b), respectively. In (b), the different Doppler-shift components ( $\mathrm{f}$ : fast, $\mathrm{r}$ : reduced, and s : slow) are indicated by the arrows. 


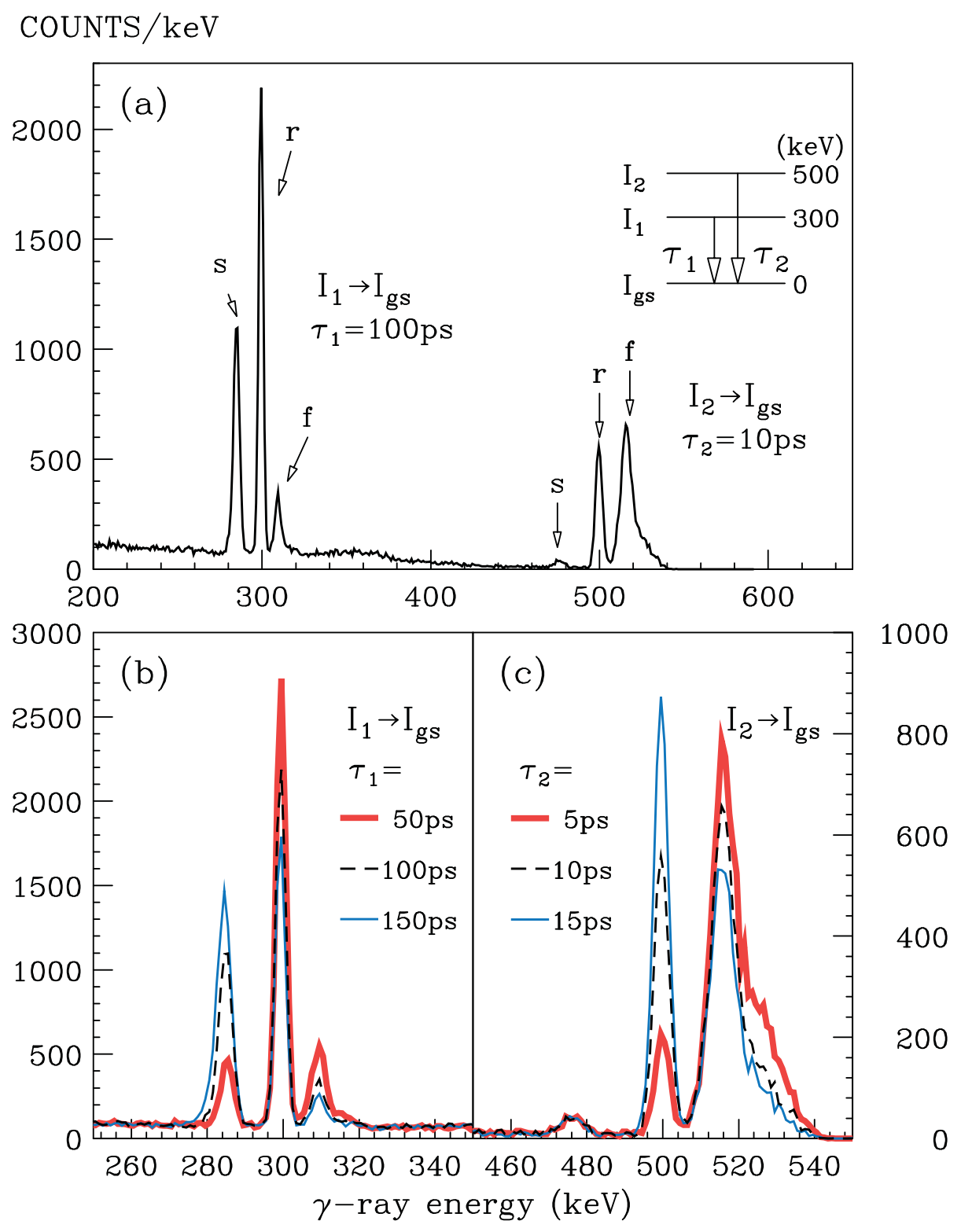

Figure 12: Simulated spectra are shown for the double recoil distance measurement with a distance setting $d_{1}=1 \mathrm{~mm}$ and $d_{2}=10 \mathrm{~mm}$. The arrows indicate the different Doppler-shift components (f : fast, $\mathrm{r}$ : reduced, and s : slow). In (a), mean lifetimes of 100 ps and 10 ps are assumed for the first $\left(I_{1}\right)$ and second $\left(I_{2}\right)$ excited states, respectively, while they are varied in (b) and (c). The bottom panels focus on each transition and the lifetimes for the $\left(I_{1}, I_{2}\right)$ states are changed to (50 ps, 5 ps) (shown in thick red solid curves), (100 ps, 10 ps) (black dashed), and (150 ps, $15 \mathrm{ps)}$ (blue solid), respectively. 


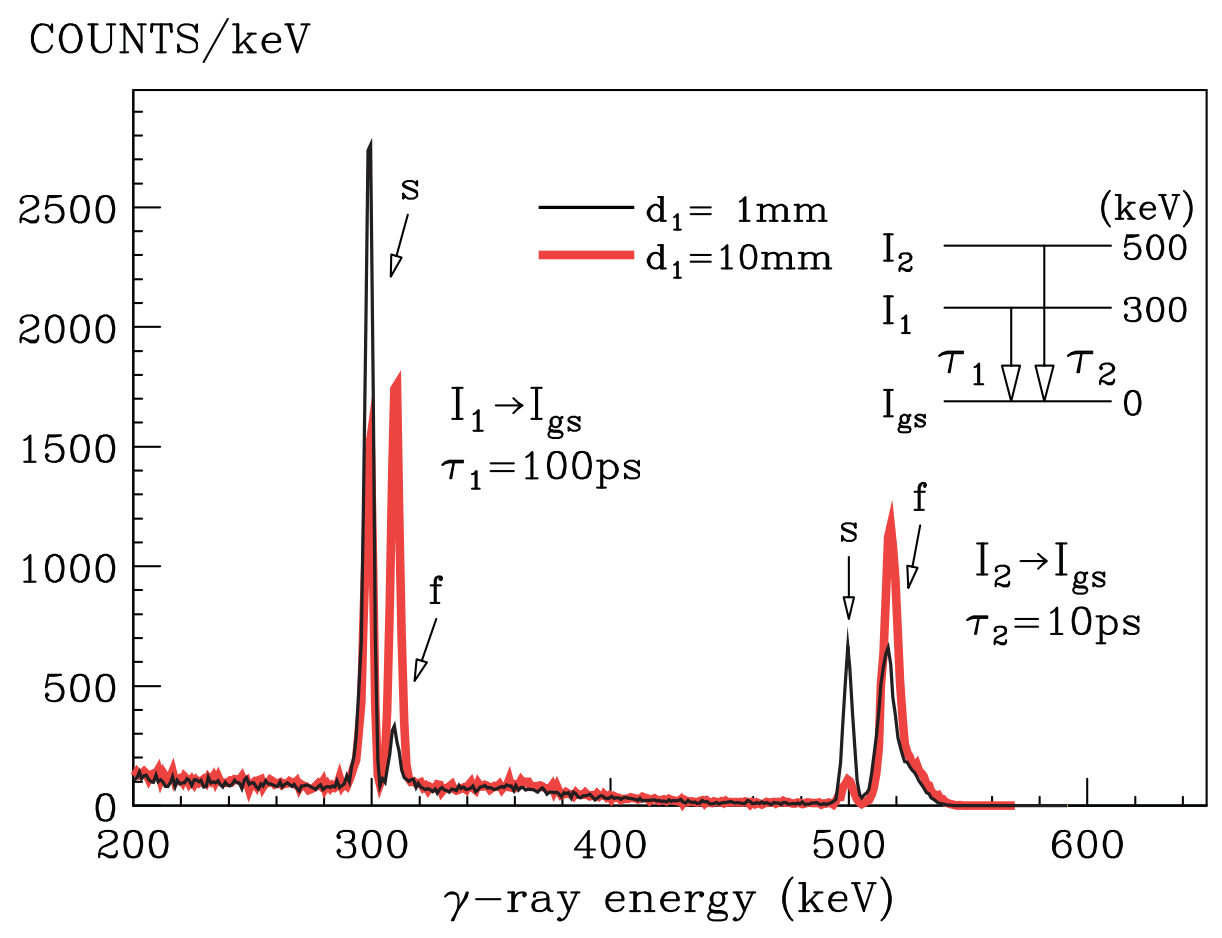

Figure 13: Simulated spectra are shown for the two-foil recoil-distance setup with the targetdegrader separation $\mathrm{d}_{1}=1 \mathrm{~mm}$ (black solid) and $10 \mathrm{~mm}$ (red bold). 


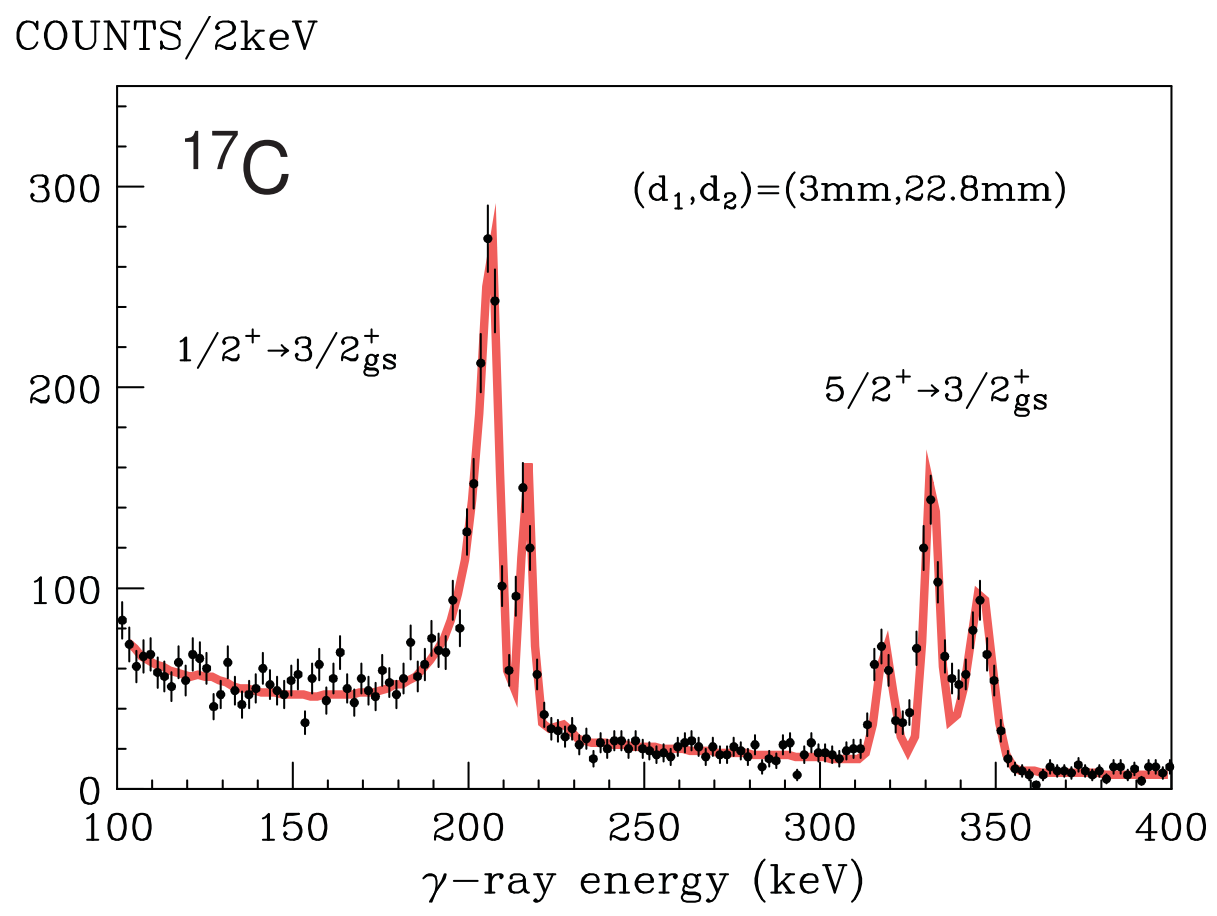

Figure 14: Measured spectrum for the ${ }^{17} \mathrm{C}$ isotope with the TRIPLEX device. Data (black points) are shown with error bars and compared to the simulated curve (red). See also the caption of Fig. 1. 
COUNTS/2keV

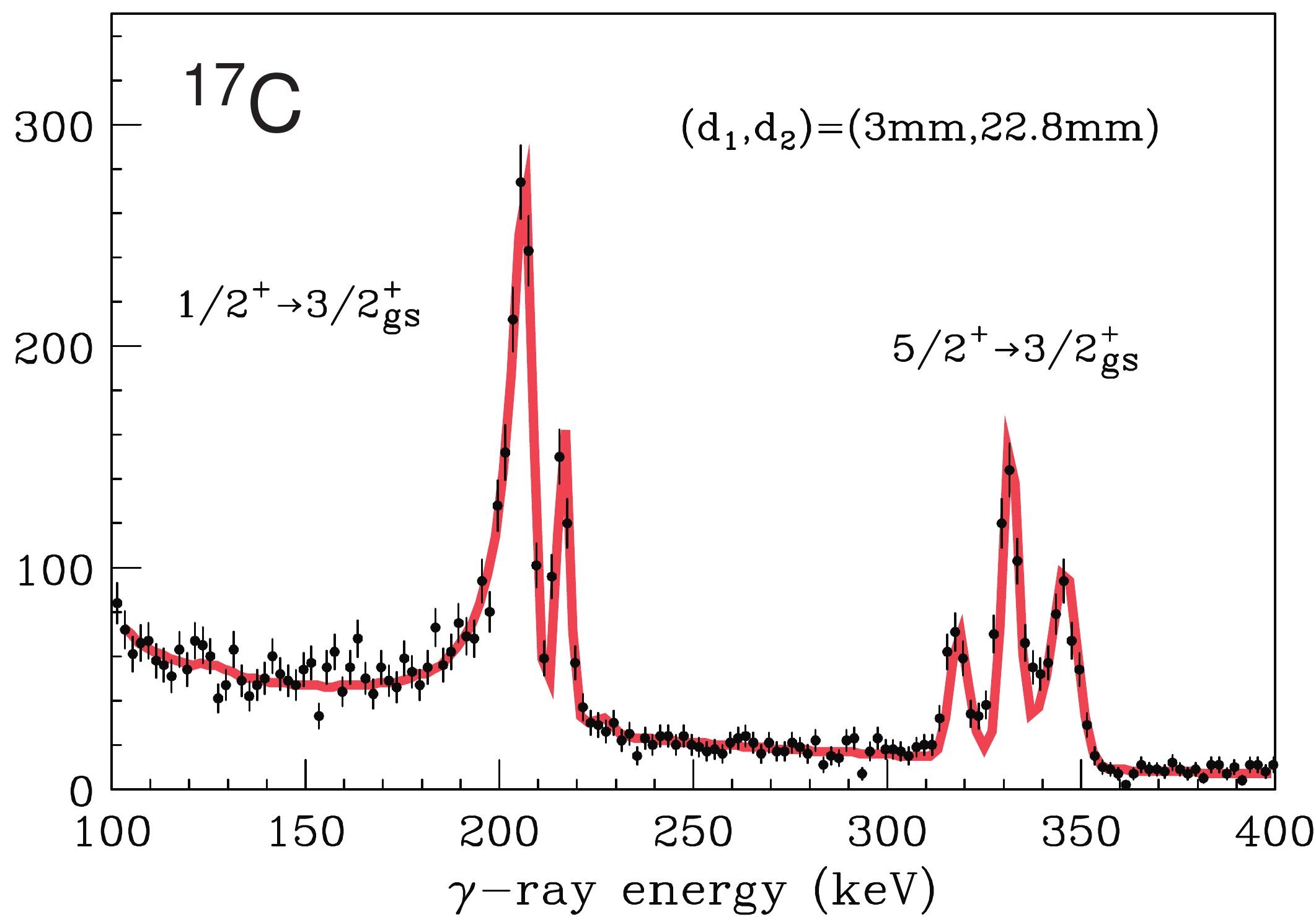

